\title{
Complexity of plastic instability in amorphous solids: Insights from spatiotemporal evolution of vibrational modes ${ }^{\star \star \star}$
}

\author{
J. Yang ${ }^{1}$, J. Duan ${ }^{1,2}$, Y.J. Wang ${ }^{1,2}$, and M.Q. Jiang ${ }^{1,2, a}$ \\ 1 State Key Laboratory of Nonlinear Mechanics, Institute of Mechanics, Chinese Academy of Sciences, Beijing 100190, China \\ 2 School of Engineering Science, University of Chinese Academy of Sciences, Beijing 101408, China
}

\author{
Received 5 July 2020 / Received in final form 15 August 2020 / Accepted 19 August 2020 \\ Published online: 11 September 2020 \\ (c) EDP Sciences / Società Italiana di Fisica / Springer-Verlag GmbH Germany, part of Springer Nature, \\ 2020
}

\begin{abstract}
It has been accepted that low-frequency vibrational modes are causally correlated to fundamental plastic rearrangement events in amorphous solids, irrespective of the structural details. But the mode-event relationship is far from clear. In this work, we carry out case studies using atomistic simulations of a three-dimensional $\mathrm{Cu}_{50} \mathrm{Zr}_{50}$ model glass under athermal, quasistatic shear. We focus on the first four plastic events, and carefully trace the spatiotemporal evolution of the associated low-frequency normal modes with applied shear strain. We reveal that these low-frequency modes get highly entangled with each other, from which the critical mode emerges spontaneously to predict a shear transformation event. But the detailed emergence picture is event by event and shear-protocol dependent, even for the first plastic event. This demonstrates that the instability of a plastic event is a result of extremely complex multiple-path choice or competition, and there is a strong, elastic interaction among neighboring instability events. At last, the generality of the present findings is shown to be applicable to covalent-bonded glasses.
\end{abstract}

\section{Introduction}

How crystalline solids deform plastically is fundamentally clear, thanks to extensive studies of a finite number of crystal types [1-3]. Under external stimuli, plastic arrangements always initiate around lattice defects such as dislocations and grain boundaries. Amorphous solids, by contrast, can exhibit an infinite variety of configurations where lattice defects are entirely lost [4]. In this case, elementary plastic events become elusive, and identifying their structural fingerprints poses a formidable challenge. Over the past decades, numerous efforts [5-11] have been made to define various predictors for amorphous plasticity. Pure structural parameters have proven to be distantly related to plastic rearrangements. But physical quantities involving dynamic properties or high-order structural information offer enhanced predictability.

Elasticity is a material property, intermediate between structure and plasticity, which can act as a middle way

\footnotetext{
* Contribution to the Topical Issue "Disordered, NonEquilibrium Systems: From Supercooled Liquids to Amorphous Solids" edited by Marco Baity Jesi, Yuliang Jin, Elijah Flenner, Marisa A. Frechero, Gustavo A. Appignanesi.

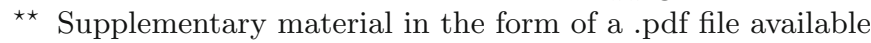
from the Journal web page at

https://doi.org/10.1140/epje/i2020-11983-6

a e-mail: mqjiang@imech.ac.cn
}

of predicting plasticity [12-21]. From a potential energy landscape perspective, elasticity and plasticity are strictly distinguished. The elasticity reflects the features of curvature of landscape basins, whereas the plasticity is barrier hopping or vanishing of inter-basins. But intuition tells us, it is easier to hop out of a relatively flat, shallow basin. This activation process can be understood as the saddlenode bifurcation of landscape induced by shear $[12,22]$. To predict such bifurcation, Maloney and Lemaître [13] have constructed the decomposition of elastic deformation in the vibrational space, yielding

$$
\frac{\mathrm{d} \mathbf{r}}{\mathrm{d} \gamma}=-\sum_{m} \frac{\zeta_{m}}{\omega_{m}^{2}} \boldsymbol{\Psi}_{m}
$$

where $\mathrm{d} \mathbf{r} / \mathrm{d} \gamma$ is the nonaffine displacement field, i.e., the change of the system's configuration $\mathbf{r}$ with applied shear strain $\gamma, \omega_{m}$ is the frequency of the vibrational normal modes $\boldsymbol{\Psi}_{m}$, and $\zeta_{m}$ is the mode decomposition of the nonaffine force field. Equation (1) predicts that, if the frequency of a mode goes to zero, this critical mode must dominate the direction of $\mathrm{d} \mathbf{r} / \mathrm{d} \gamma$ whose length will diverge simultaneously. Such a divergence accounts for the breakdown of eq. (1) as an indicative of the onset of a plastic event. Guided by the low-frequency critical modes or their derivatives, one can identify structural "soft spots" where plastic events sprout with a high propensity [18,23-26]; the triggering strain of plastic events can be predicted [27]. 
In this work, we do not straightly aim at the prediction of amorphous plasticity by defining any modederived indicator. In contrast, we restrict our attention to the spatiotemporal evolution of vibrational modes associated with plastic events, which has so far not been studied in details. The evolution of modes per se is the dynamic response of hyper-dimensional energy landscape to external loading. To highlight the essential physics, we purposely focus on the first four plastic events in a model glass, and carefully trace the event-related low-frequency modes during shear straining. This belongs to case studies, but vividly portraying extreme complexity of plastic instability events in amorphous solids, from which we can gain some useful insights into amorphous plasticity.

\section{Atomistic simulations}

A well-studied three-dimensional $(3 \mathrm{D}) \mathrm{Cu}_{50} \mathrm{Zr}_{50}$ model glass [28-30] is adopted as a representative of general amorphous solids, which contains 19652 atoms interacting with a Finnis-Sinclair-type embedded-atom method potential [31]. We prepare this model glass from its melting state, which has been equilibrated at $2000 \mathrm{~K}$ for $2 \mathrm{~ns}$ that is much longer than its $\alpha$-relaxation time. Then the equilibrated liquid is quenched to $0 \mathrm{~K}$ glass configuration with a cooling rate of $10^{10} \mathrm{~K} / \mathrm{s}$. An additional sub- $T_{g}$ annealing at $700 \mathrm{~K}$ is performed for $60 \mathrm{~ns}$ to accelerate the aging dynamics, which drives the inherent structure (IS) to the deeper basin on the energy landscape. The prepared stable glass is of distinguishable low-frequency modes and therefore facilitates the clarification of the contribution of versatile modes to the displacement field.

We perform the athermal quasistatic shear (AQS) atomistic simulations to harvest plastic events triggered by shear strain. A simple shear is applied on the glass with a very small strain step $\delta \gamma=10^{-5}$, and the LeesEdwards boundary conditions are used throughout. During the AQS protocol, the glass configuration $\mathbf{r}$ always resides in an IS of potential energy $U(\mathbf{r}, \gamma)$. For the IS at a given strain, the normal modes $\boldsymbol{\Psi}_{m}$ and corresponding $\omega_{m}$ are computed through the exact diagonalization of the Hessian matrix $\mathbf{H}=\partial U / \partial \mathbf{r} \partial \mathbf{r}$ with a cutoff radius of $7.6 \AA$. The nonaffine displacement $\delta \mathbf{r}$ during each $\delta \gamma$ is calculated as the real displacement minus the macroscopic affine portion; here $\delta \mathbf{r}$ can be either elastic or plastic.

\section{Results and discussion}

Figure 1 shows the first four plastic events, manifesting as the four stress drops separated by linear elastic segments. The inset gives the typical stress vs. strain trajectory under AQS, which is well resolved by the prescribed strain step $\delta \gamma=10^{-5}$. It can be seen that the first four events occur at very early stage of straining, far below the global yield point. Their onset strains $\gamma_{c}$ are $0.253 \%, 0.277 \%$, $0.295 \%$ and $0.534 \%$, respectively. Each event is complete in an individual strain step. Such low triggering strains

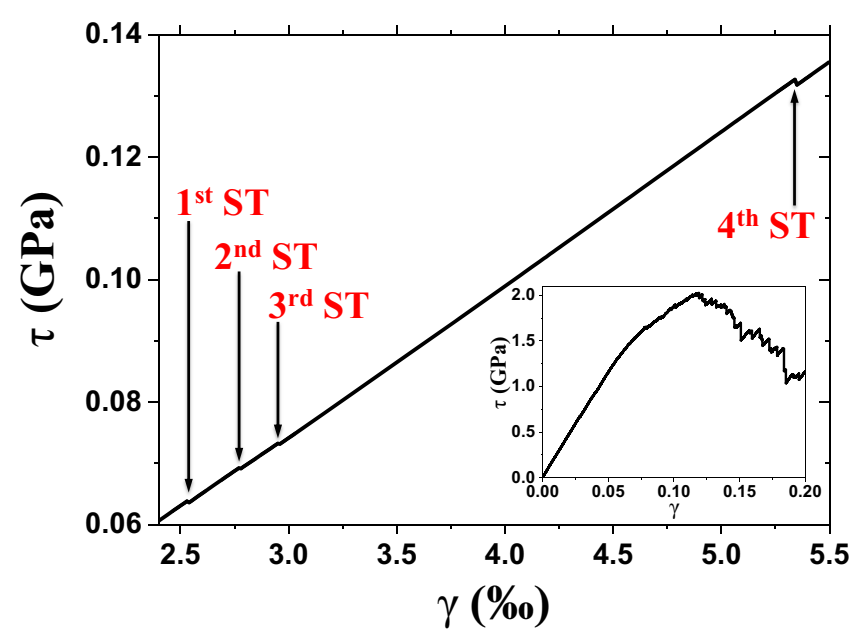

Fig. 1. The first four plastic ST events indicated as four stress drops in the AQS stress vs. strain trajectory. Inset: the entire trajectory beyond the global yield.

imply that, in the AQS limit, the studied glass resides in a marginal stable state [26,32-34]. The IS of glass is rather rough and hierarchical, hence any tiny basin could be sampled during straining. This is further evidenced by the real-space configurations of the four events in 3D; see fig. S1 in the Electronic Supplementary Material (ESM). We find that these events belong to localized shear transformations (STs) occurred at distinct spatial positions. It must be addressed that such spatial separation of plastic events benefits from the very deep basin of the initial sample. Otherwise events' overlap in space possibly occurs. Their ST nature is supported by the atomic shear-strain correlation that they induce; see fig. S2 in the ESM, showing the quadrupolar pattern like an Eshelby inclusion [35]. The four ST events look spatiotemporally isolated. We therefore take them as case studies to explore their plastic instabilities in the mode space.

Figure 2(a) plots the stress difference $\delta \tau=$ $\tau(\gamma)-\tau(\gamma-\delta \gamma)$ as a function of $\gamma$. This plot to some extent reflects the change of shear modulus with strain, in which the four ST events are magnified as precipitous drops of $\delta \tau$. The lowest-frequency ten modes $\boldsymbol{\Psi}_{m}$ are calculated for the initially unstrained state $\gamma_{0}$, and they are numbered as modes $1,2, \ldots$, and 10 in increasing order of their frequencies $\omega_{m}$. We calculate the participation ratio of the ten modes, and find that modes 1 and 2 belong to quasilocalized modes that should obey the non-Debye $\omega_{m}^{4}$ scaling law [36-38], while the others are extended Debye modes. By the spatial projection of $\boldsymbol{\Psi}_{m}$ (see sect. 3 in the ESM), we carefully trace these modes during the straining process, particularly when they are crossing/anti-crossing or pass through plastic events. Figure 2(b) gives the evolution of the frequencies $\omega_{m}$ of the ten modes from the initial state to the onset of the fourth event. It can be seen that, preceding each stress drop (or ST) event, there always emerges a critical mode $\boldsymbol{\Psi}_{*}$ with its frequency diverging towards zero $\left(\omega_{*} \rightarrow 0\right)$ at $\gamma_{c}$. This is consistent with previous reports $[13,17$, $23]$. The critical modes are $1,2,3$ and 6 , respectively, 


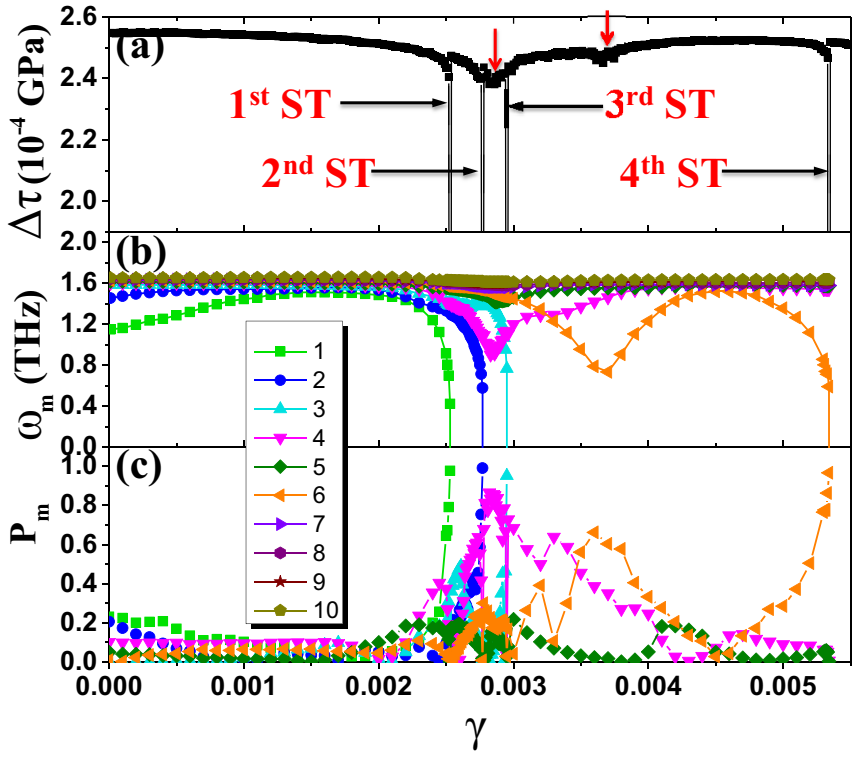

Fig. 2. (a) The stress difference $\delta \tau=\tau(\gamma)-\tau(\gamma-\delta \gamma)$, (b) the lowest ten mode frequencies $\omega_{m}$ and (c) the projection $P_{m}$ of the nonaffine displacement field $\delta \mathbf{r}(\gamma \rightarrow \gamma+\delta \gamma)$ onto the modes $\boldsymbol{\Psi}_{m}(\gamma)$ as a function of applied strain $\gamma$.

corresponding to the first four events. However, these ST events are not as simple as they look at first glance. We notice that modes 4 and 5 are also stirred by the applied shear straining. Six low-frequency modes thereof participate in the occurrence of the first four ST events. The frequency evolution of these modes is highly straindependent, which essentially reflects how the glass selects the deformation paths to release the plastic ST events.

To quantify this instability-path selectivity, we project the nonaffine displacement field $\delta \mathbf{r}$ onto the vibrational mode $\boldsymbol{\Psi}_{m}$, here $\boldsymbol{\Psi}_{m}$ is calculated at the start strain point of $\delta \mathbf{r}$. The projection parameter is defined as

$$
P_{m}=\boldsymbol{\Psi}_{m} \cdot \delta \mathbf{r} /|\delta \mathbf{r}| .
$$

where $0 \leq P_{m} \leq 1$. The larger the $P_{m}$ value is, the higher the directional consistence between $\delta \mathbf{r}$ and $\boldsymbol{\Psi}_{m}$. Therefore, this defined parameter can quantitatively measure the instantaneous contribution of each mode to the nonaffine deformation. Figure 2(c) calculates the strain evolution of the $P_{m}$ values for the lowest six modes: $\boldsymbol{\Psi}_{m}, m=$ $1,2, \ldots, 6$. We observe that, corresponding to the sudden drop of $\omega_{*}$ to 0 at $\gamma_{c}$, the contribution $P_{*}$ of the critical mode $\boldsymbol{\Psi}_{*}$ will sharply rise close to 1 , which also indicates the onset of a plastic event but from a spatial viewpoint. The observed spatiotemporal consonance reveals that plastic instabilities follow the $\boldsymbol{\Psi}_{*}$-controlled minimum energy path. It is noted that the critical modes $\boldsymbol{\Psi}_{*}$ can even dominate the configuration $\delta \mathbf{r}$ of the entire plastic events next to them, since all $P_{*}\left(\gamma_{c}\right) \rightarrow 1$. This feature should be intrinsic to individual ST events, but not suitable for the avalanche plastic events involving multiple STs [13,39].

However, far away from the critical instability points, the situation becomes much more complex. $P_{m}$ is more susceptible than $\omega_{m}$ to reflect the complexity of defor- mation paths. We notice that, at some strain points, the maximum $P_{m}$ does not necessarily correspond to the mode with the minimum $\omega_{m}$. This might result from the additional contribution $\zeta_{m}$ of the nonaffine force [7,27], which in turn confirms that the critical modes only dominate the configurational change close to plastic instabilities. We also find that the $P_{m}$ values could be almost identical for different frequencies $\omega_{m}$. This means that the glass arrives at a fork in the deformation path. More importantly, the path instabilities will compete or interfere with each other. For example, after the 2nd ST, the path instability along the mode 4 is surpassed by that along the mode 3 (leading to the 3rd ST), and thus becomes stable again. The revival of the mode 4 afterwards impedes the first instability along the mode 6 , although the second instability of the latter leads to the 4th ST. The two terminations of path instabilities exactly correspond to the frequency kinks of modes 4 and 6 , respectively, in fig. 2(b). This accounts for the two abortive stress drops $\delta \tau$ as marked by red arrows in fig. 2(a). These results reveal a strong, elastic interaction among these spatiotemporally-isolated plastic events, which is reminiscent of the elastic avalanches reported recently $[34,40]$. The event-event interaction observed here benefits from the continual, across-event trace of vibrational modes and nonaffine displacements.

Next, we explore the spatial patterns of both deformation fields $\delta \mathbf{r}$ and vibrational modes $\boldsymbol{\Psi}_{m}$, which are projected on the shear $(e . g ., x-y)$ plane. But the atoms are chosen from a proper slab that resides in the $x-y$ plane and has a thickness of $6 \AA$ along the $z$ direction. The identical slabs are used for a plastic event and its associated vibrational modes. From fig. 2, we know that the vibrational modes related to plastic events are strongly strain-dependent. It is further confirmed by fig. 3 which shows that the initial pattern of the critical mode is totally different from its final pattern. The latter has a configurational consistence close to 1 with its corresponding plastic event, see fig. 2(c). The four critical modes at $\gamma_{c}$ are of typical quasilocalized character, but their initial feature at $\gamma_{0}$ can be either quasilocalized (modes 1 and 2 ) or extended (modes 3 and 6 ).

We will purposely examine specific strain points, in order to visualize the interaction of vibrational modes and their relationship with upcoming plastic events. The 1st ST event is a perfect case, since it seems to only link with the mode 1 . Figure 4 shows that this is not so straightforward - before the strain of $0.220 \%$, the mode $\boldsymbol{\Psi}_{2}$ (Row (c)) looks more similar to the $\delta \mathbf{r}$ during the 1st ST (see fig. S4(a) in the ESM). Keep in mind, at that strain stage, this similarity cannot be detected, because we do not know the configuration of the 1st ST. In addition, the frequency $\omega_{2}$ of the mode $\boldsymbol{\Psi}_{2}$ does not show any anomaly before the $0.220 \%$ strain (see fig. 2(b)). At the $0.220 \%$ strain, the modes $\boldsymbol{\Psi}_{1}$ (Row (b)) and $\boldsymbol{\Psi}_{2}$ are almost the same, indicating an entanglement of deformation paths. After this fork point, the spatial pattern of the mode $\boldsymbol{\Psi}_{1}$ gradually points to the 1st ST's configuration, whereas the mode $\boldsymbol{\Psi}_{2}$ evolves along other directions. Later, the $\boldsymbol{\Psi}_{2}$ leads to the 2nd ST, but at that time we do not know. The path transfer from the mode 2 to 1 explains why the lowest $\omega_{1}$ 

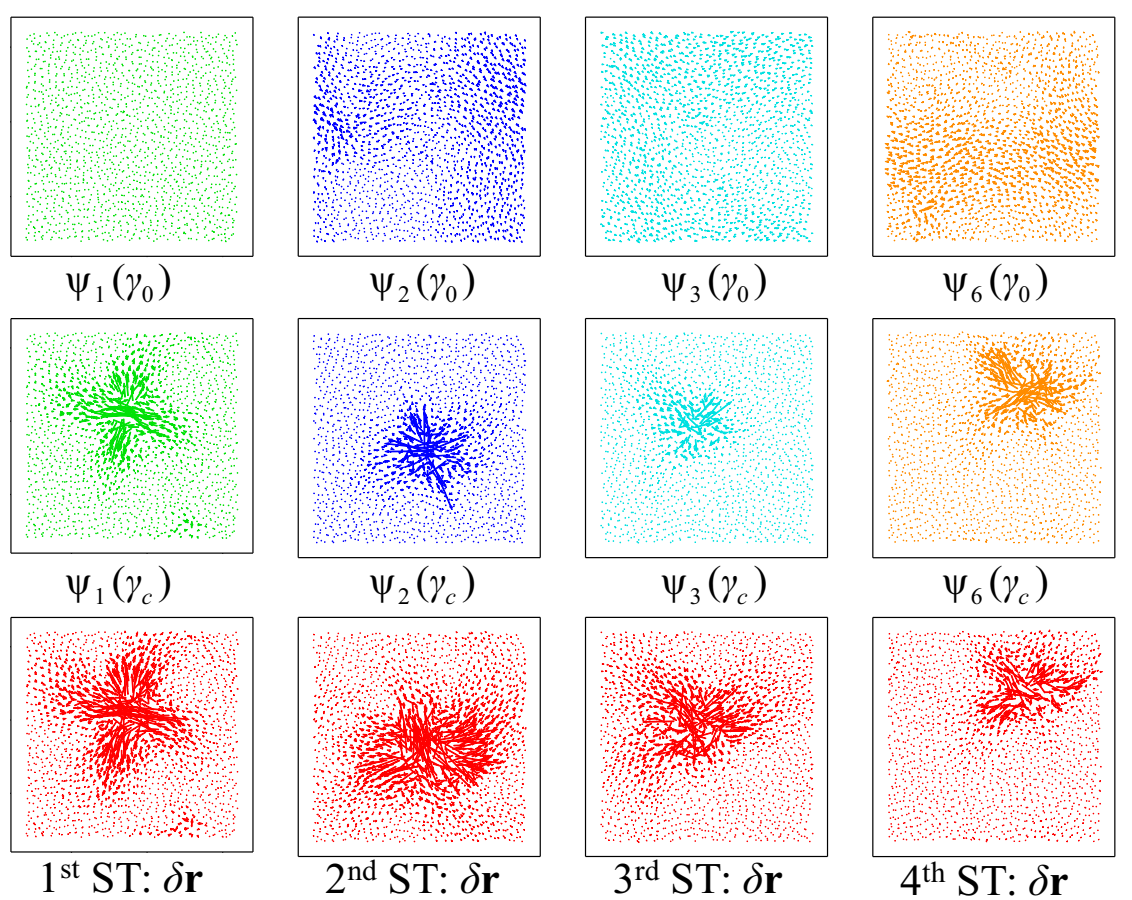

Fig. 3. The initial patterns (upper panel) of critical modes and their final patterns (middle panel) at the critical instability strains. Lower panel: the nonaffine displacement fields during the plastic events corresponding to these critical modes.

\section{(a) $\delta \mathbf{r}$}

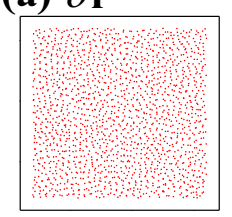

(b) $\psi_{1}$

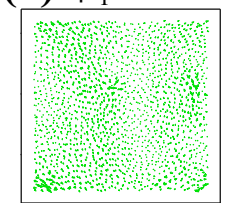

(c) $\psi_{2}$

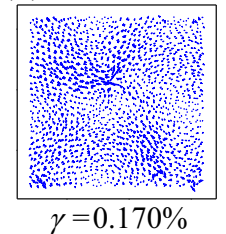

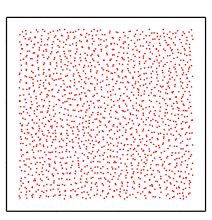
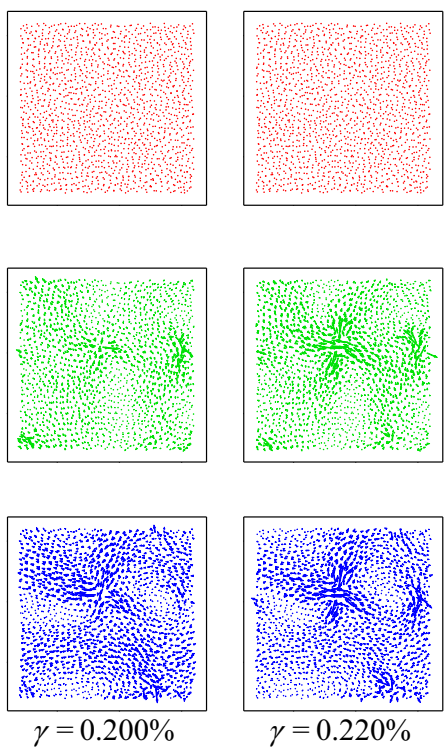
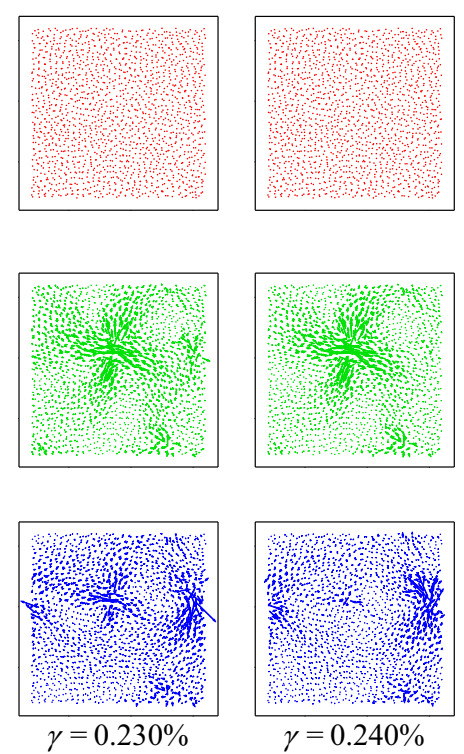
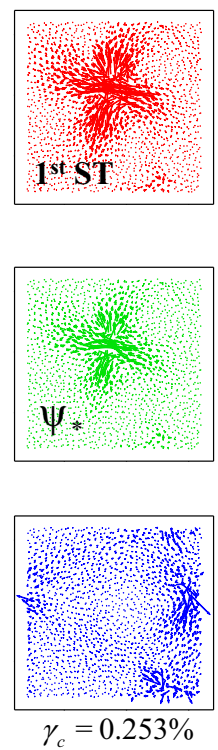

Fig. 4. A cast study showing the emergence of the 1st ST event associated with vibrational modes. Row (a): the nonaffine displacement field $\delta \mathbf{r}$. Row (b): the critical mode $\left(\boldsymbol{\Psi}_{*}=\boldsymbol{\Psi}_{1}\right)$ of the 1st ST event. Row (c): the mode $\boldsymbol{\Psi}_{2}$. Note that $\delta \mathbf{r}$ occurs in the strain step $\delta \gamma$ just next to the strain $\gamma$ at which the modes are calculated.

first shift upwards to the $\omega_{2}$ and then decreases sharply to zero (see fig. 2(b)). It seems that the mode 1 is not passively hybridized by the mode 2 . Instead, the former is actively regulated by the latter and becomes the critical destabilizing mode thereof.

It must be pointed out that the elastic configurational change $\delta \mathbf{r}$ is negligibly small, and keeps almost unchanged as compared to $\delta \mathbf{r}$ of plastic events (Row (a)). Therefore, the path selection detected in the mode space is barely perceptible in the real space. This suggests that normal modes, as a set of base directions of nonaffine displacements, provide an effective probe into the deformation paths before plastic instabilities.

The case studies of the 2nd, 3rd, and 4th ST events are presented, respectively, in figs. S4(b)-(d) and S5-S7 of the ESM. These events show more complex than the 
(a)

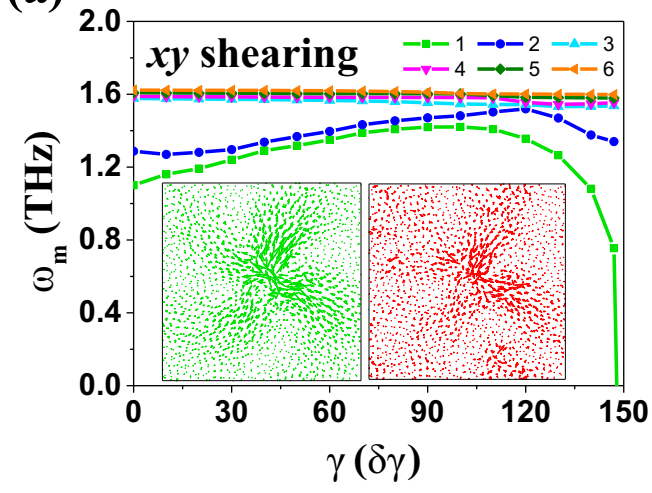

(c)

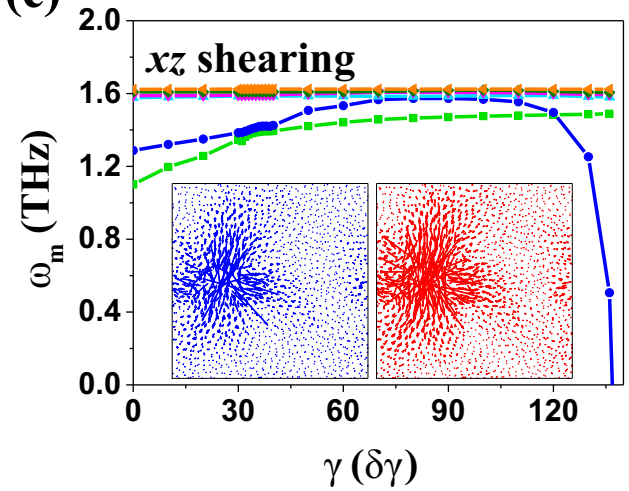

(e)

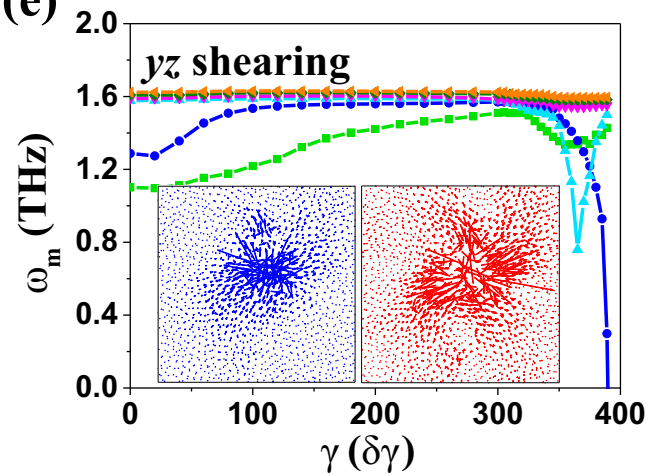

(b)

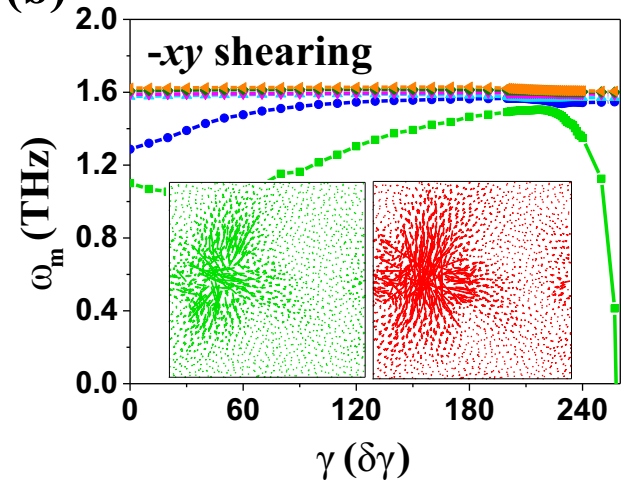

(d)

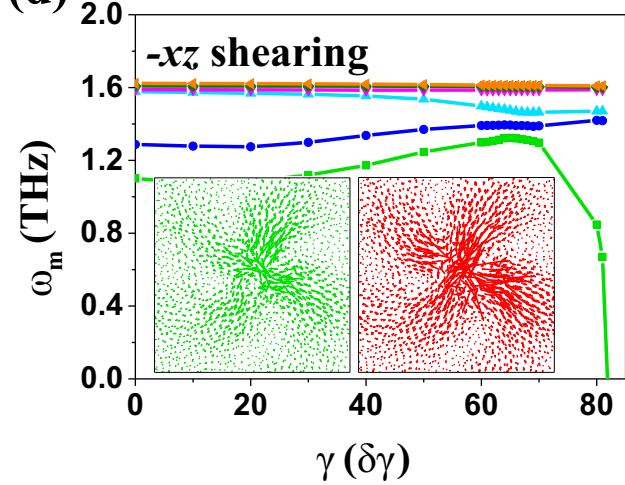

(f)

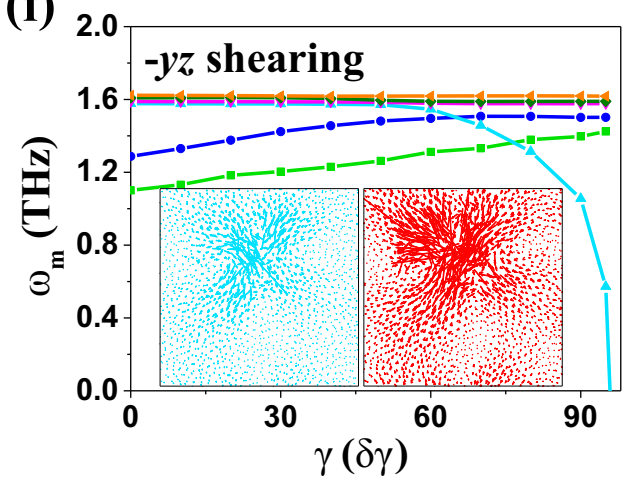

Fig. 5. The lowest six-mode frequencies as a function of applied shear strain along the (a) $x y$, (b) $-x y$, (c) $x z,(d)-x z,(\mathrm{e})$ $y z$ and (f) $-y z$ directions. The critical mode emerges from these low-frequency modes, indicating the onset of the first plastic event. Inset: the critical mode at $\gamma_{c}$ and the nonaffine rearrangement during the first event.

1st one, since at least three modes are observed to participate in their respective instabilities. For the 2nd ST event, the participant modes are 2 (critical mode), 3 and 4 . For the 3rd ST, the modes are 3 (critical mode), 5 and 6 . For the 4 th ST, the modes are 4, 5 and 6 (critical mode). The three-mode competition can easily incur a trident fork of deformation paths, which can be seen at $\gamma=0.250 \%$ in fig. S5 or $\gamma=0.450 \%$ in fig. S7. The multiple-path choice of the plastic event and strong interaction of neighboring events thereof can be also observed. But the details related to each plastic event are case by case, showing the emergent universality [41].

Finally, we study the shear-protocol dependence of plastic events, and its importance has been addressed recently $[7,27]$. We prepare a cube-shaped $\mathrm{Cu}_{50} \mathrm{Zr}_{50}$ glass that is apparently isotropic, and perform AQS simulations on it by simple shear along the $x y,-x y, x z,-x z$, $y z$ and $-y z$ directions. We trace the lowest-frequency six modes and see which mode induces the first plastic ST event. The results are shown in fig. 5. It can be seen that different shearing protocols lead to different manners in which the critical mode emerges. As a result, the critical strains triggering the first plastic event are entirely different, reflecting the difficulty of activation of incipient plasticity. Among the six critical modes, three evolves from the lowest-frequency mode 1 ; two from the mode 2; one from the mode 3 . However, it is interesting to find that different deformation paths probably lead to similar or identical plastic events in space. For example, the first event for the $x y$ shearing has a configurational consis- 
tence of $\sim 0.86$ with that in the $-x z$ direction, although both of them undergo different strain-evolution processes. A similar phenomenon is also observed for the $-x y$ and $x z$ shearings: the two plastic events are of configurational consistence close to 1 . In this situation, different deformation paths can even follow different modes (1 and 2), but leading to identical plastic events at different trigger strains.

\section{Conclusions}

We conclude this work with some insights beyond the previous knowledge of amorphous plasticity. The new insights benefit from both the multiple-mode tracing of plastic events and the across-event tracing of vibrational modes. First, we realize that the spatiotemporal entanglement of neighboring modes, i.e., so-called low-frequency hybridization, is not meaningless far away from plastic events; on the contrary, it is a necessary or natural process in which the glass actively chooses the deformation paths on the energy landscape to reach plastic instabilities. Second, the critical or destabilizing mode hybridizes not only with background plane waves, but also with other destabilizing modes leading to plastic events. This results in a strong, elastic interaction among neighboring plastic events even at the very early stage of deformation. The occurrence of a plastic event will in turn reconstruct the low-frequency modes and thus affect the next event. Such mode-event interplay is the core of amorphous elastoplastic models $[42,43]$. Thirdly, the strain evolution of normal modes shown here, to some extent, reflects the information on the anharmonicities of the potential energy landscape. The importance of the latter has been corroborated recently $[10,44]$, where nonlinear modes can be defined by expanding the landscape to higher-order approximation around its minimum. However, from our opinion, neither normal nor nonlinear modes have the power to predict a plastic event at a strain distance very far away from the instability onset, although the latter offers higher accuracy. It remains greater challenging to achieve the across-event prediction for both types of modes. We therefore suggest that statistic description of modes, normal or nonlinear, may be more viable to provide a probability prediction of plastic events, as done in refs. [23,26].

Last but not least, we carry out extra simulations on a new model of the $\mathrm{Pd}_{80} \mathrm{Si}_{20}$ glass; see figs. $\mathrm{S} 8$ and $\mathrm{S} 9$ in the ESM. This model is a metal-metalloid alloy that is very different from the $\mathrm{Cu}_{50} \mathrm{Zr}_{50}$ glass, a typical metal-metal system. In this way, the generality of the present findings is shown to be applicable to either metallic-bonded or covalent-bonded glasses.

This work was supported by the National Natural Science Foundation of China (NSFC) Basic Science Center for "Multiscale Problems in Nonlinear Mechanics" (Grant No. 11988102), the NSFC (Grant No. 11972345, and No. 11790292), and the Youth Innovation Promotion Association of Chinese Academy of Sciences (Grant No. Y201704).

\section{Author contribution statement}

M.Q. Jiang conceived the idea of this work. J. Yang and J. Duan performed the simulations. M.Q. Jiang and Y.J. Wang analyzed the data and wrote the manuscript.

Publisher's Note The EPJ Publishers remain neutral with regard to jurisdictional claims in published maps and institutional affiliations.

\section{References}

1. U.F. Kocks, A.S. Argon, M.F. Ashby, Thermodynamics and Kinetics of Slip (Pergamon Press, New York, Oxford, 1975).

2. X. Li, Y. Wei, L. Lu, K. Lu, H. Gao, Nature 464, 877 (2010).

3. H. Song, D. Dimiduk, S. Papanikolaou, Phys. Rev. Lett. 122, 178001 (2019).

4. P.G. Debenedetti, F.H. Stillinger, Nature 410, 259 (2001).

5. Y.Z. Lu, M.Q. Jiang, X. Lu, Z.X. Qin, Y.J. Huang, J. Shen, Phys. Rev. Appl. 9, 014023 (2018).

6. S. Patinet, D. Vandembroucq, M.L. Falk, Phys. Rev. Lett. 117, 045501 (2016).

7. Z. Schwartzman-Nowik, E. Lerner, E. Bouchbinder, Phys. Rev. E 99, 060601 (2019).

8. E.D. Cubuk et al., Phys. Rev. Lett. 114, 108001 (2015).

9. X. Yang, R. Liu, M. Yang, W.-H. Wang, K. Chen, Phys. Rev. Lett. 116, 2388003 (2016).

10. J. Zylberg, E. Lerner, Y. Bar-Sinai, E. Bouchbinder, Proc. Natl. Acad. Sci. U.S.A. 114, 7289 (2017).

11. R. Shi, P. Xiao, R. Yang, Y. Bai, Int. J. Solids Struct. 191-192, 363 (2020).

12. D.L. Malandro, D.J. Lacks, Phys. Rev. Lett. 81, 5576 (1998).

13. C. Maloney, A. Lemaître, Phys. Rev. Lett. 93, 195501 (2004).

14. C.E. Maloney, A. Lemaitre, Phys. Rev. E 74, 016118 (2006).

15. M. Tsamados, A. Tanguy, C. Goldenberg, J.-L. Barrat, Phys. Rev. E 80, 026112 (2009).

16. S. Karmakar, A. Lemaître, E. Lerner, I. Procaccia, Phys. Rev. Lett. 104, 215502 (2010).

17. R. Dasgupta, S. Karmakar, I. Procaccia, Phys. Rev. Lett. 108, 075701 (2012).

18. J. Rottler, S.S. Schoenholz, A.J. Liu, Phys. Rev. E 89, 042304 (2014).

19. M. Ovaska, A. Lehtinen, M.J. Alava, L. Laurson, S. Zapperi, Phys. Rev. Lett. 119, 265501 (2017).

20. C.-C. Vu, D. Amitrano, O. Plé, J. Weiss, Phys. Rev. Lett. 122, 015502 (2019).

21. J. Ding, Y.-Q. Cheng, H. Sheng, M. Asta, R.O. Ritchie, E. Ma, Nat. Commun. 7, 13733 (2016).

22. N. Xu, V. Vitelli, A.J. Liu, S.R. Nagel, EPL 90, 56001 (2010).

23. M.L. Manning, A.J. Liu, Phys. Rev. Lett. 107, 108302 (2011).

24. J. Ding, S. Patinet, M.L. Falk, Y.Q. Cheng, E. Ma, Proc. Natl. Acad. Sci. U.S.A. 111, 14052 (2014).

25. H. Tong, N. Xu, Phys. Rev. E 90, 010401 (2014).

26. H. Tong, H. Hu, P. Tan, N. Xu, H. Tanaka, Phys. Rev. Lett. 122, 215502 (2019). 
27. B. Xu, M.L. Falk, J.F. Li, L.T. Kong, Phys. Rev. Lett. 120, 125503 (2018).

28. J. Yang, Y.-J. Wang, E. Ma, A. Zaccone, L.H. Dai, M.Q. Jiang, Phys. Rev. Lett. 122, 015501 (2019).

29. D. Wei, J. Yang, M.Q. Jiang, B.-C. Wei, Y.-J. Wang, L.-H. Dai, Phys. Rev. B 99, 014115 (2019).

30. D. Wei et al., J. Chem. Phys. 150, 114502 (2019).

31. M.I. Mendelev, M.J. Kramer, R.T. Ott, D.J. Sordelet, D. Yagodin, P. Popel, Philos. Mag. 89, 967 (2009).

32. P. Charbonneau, J. Kurchan, G. Parisi, P. Urbani, F. Zamponi, Nat. Commun. 5, 3275 (2014).

33. P. Cao, M.P. Short, S. Yip, Proc. Natl. Acad. Sci. U.S.A. 116, 18790 (2019)

34. B. Shang, P. Guan, J.-L. Barrat, Proc. Natl. Acad. Sci. U.S.A. 117, 86 (2019).

35. J.D. Eshelby, Proc. R. Soc. London, A 241, 376 (1957)
36. E. Lerner, G. Düring, E. Bouchbinder, Phys. Rev. Lett. 117, 035501 (2016).

37. H. Mizuno, H. Shiba, A. Ikeda, Proc. Natl. Acad. Sci. U.S.A. 114, E9767 (2017).

38. L. Wang, A. Ninarello, P. Guan, L. Berthier, G. Szamel, E. Flenner, Nat. Commun. 10, 26 (2019).

39. A. Tanguy, B. Mantisi, M. Tsamados, EPL 90, 16004 (2010).

40. S.-X. Peng et al., J. Chem. Phys. 150, 111104 (2019).

41. E.D. Cubuk et al., Science 358, 1033 (2017).

42. A. Nicolas, E.E. Ferrero, K. Martens, J.-L. Barrat, Rev. Mod. Phys. 90, 045006 (2018).

43. D.F. Castellanos, M. Zaiser, Phys. Rev. Lett. 121, 125501 (2018).

44. L. Gartner, E. Lerner, Phys. Rev. E 93, 011001(R) (2016). 